\title{
Dual Multi-kernel Discriminant Analysis for Color Face Recognition
}

Qian Liu $^{\text {a, b,* }}$, Chao Wang ${ }^{\text {a, b }}$, Xiao-yuan Jing ${ }^{\text {c }}$

a Jiangsu Key Laboratory of Meteorological Observation and Information Processing, Nanjing University of Information Science \& Technology, Nanjing, Jiangsu, China, 210044.

${ }^{\mathrm{b}}$ (Correspondence Address) School of Electronic \& Information Engineering, Nanjing University of Information Science \& Technology, Nanjing, Jiangsu, China, 210044. E-mail: lqsxld@163.com

${ }^{c}$ College of Automation, Nanjing University of Posts and Telecommunications, Nanjing, Jiangsu, China, 210023.

\section{Abstract}

With the increasing use of color images in the fields of pattern recognition, computer vision and machine learning, color face recognition technique becomes important, whose key problem is how to make full use of the color information and extract effective discriminating features. In this paper, we propose a novel nonlinear feature extraction approach for color face recognition, named dual multi-kernel discriminant analysis (DMDA), where we design a kernel selection strategy to select the optimal kernel mapping function for each color component of face images, further design a color space selection strategy to choose the most suitable space, then separately map different color components of face images into different high-dimensional kernel spaces, and finally perform multi-kernel learning and discriminant analysis not only within each component but also between different components. Experimental results in the public face recognition grand challenge (FRGC) version 2 and labeled faces in the wilds (LFW) databases illustrate that our approach outperforms several representative color face recognition methods. 


\section{Keywords}

Color face recognition, multi-kernel learning, discriminant analysis, nonlinear feature extraction, subspace learning, face recognition grand challenge (FRGC), labeled faces in the wilds (LFW).

\section{Introduction}

Color images have been widely used all over the world, and particularly they are increasingly used in the fields of pattern recognition, computer vision and machine learning, because they can offer more identifiable information than grayscale images [1-3]. Currently, the RGB color space is the most commonly used color space, and other color spaces are generally converted from the RGB space via a linear or nonlinear transformation. Fig. 1 shows a color face image and its color component images in RGB, HSV and $\mathrm{YC}_{\mathrm{b}} \mathrm{C}_{\mathrm{r}}$ color spaces. It is obvious that all color component images have similar facial contours and chiaroscuro to the color image, although they look evidently different and the contours in some color component images are fuzzy. This difference generated by colors can provide more useful information for recognition tasks. Therefore, the key problem of color face recognition technique is how to make full use of the color information and extract effective discriminating features [4-6].

Some color face recognition methods have been presented to solve this problem, which can be divided into following two categories. 


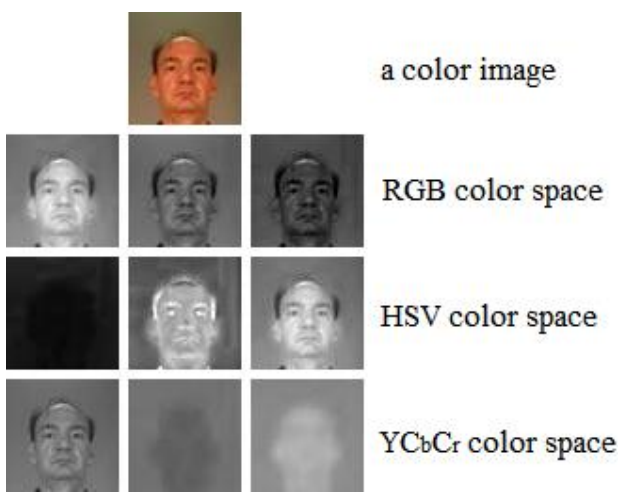

Fig. 1. A color face image and its color component images in RGB, HSV and $\mathrm{YC}_{\mathrm{b}} \mathrm{C}_{\mathrm{r}}$ color spaces, where the color face image in the first row comes from the public face recognition grand challenge (FRGC) version 2 database [29], the left, center and right images in the second row separately are the $\mathrm{R}, \mathrm{G}$ and $\mathrm{B}$ component images, the images in third row are the $\mathrm{H}, \mathrm{S}$ and $\mathrm{V}$ component images in turn, and the images in fourth row are the $\mathrm{Y}, \mathrm{C}_{\mathrm{b}}$ and $\mathrm{C}_{\mathrm{r}}$ component images in sequence.

(1) Most of color face recognition methods extract features from color images by using the linear transformation or linear projection techniques. For examples, Choi et al. [7] presented a boosting color-component feature selection framework to seek the best set of color-component features from diverse color spaces (or models). Wang et al. represented a color image as a third-order tensor and constructed a tensor discriminant color space (TDCS) model [8] to produce a discriminant color space while extracting features. In next year, the TDCS model was extended to sparse TDCS [9], which achieves a sparse color space transformation matrix and two sparse discriminant projection matrices. Shin et al. [10] applied sparse representations to multiple color components of face images, and employed score-level fusion to merge the complementary residuals obtained from different color component images. Liu [11] designed a method to obtain a compact color image representation and extract features via discriminant analysis. Zhao et al. [12] developed a two-dimensional color uncorrelated discriminant analysis method, which serially extracts discriminating 
features of $\mathrm{R}, \mathrm{G}$ and $\mathrm{B}$ components and meanwhile makes the obtained projection transformations mutually statistically orthogonal. Huang et al. [13] formalized a color image as a third-order tensor, and then presented a sparse tensor canonical correlation analysis method for feature extraction. Wu [14] used the quaternion to represent a color pixel, and applied the locality preserving projection method to the quaternion vectors of color images to extract features. Sun et al. [15] combined the R, G and B color components of a face image into one monochromic image, and developed a color image correlation similarity discriminant model to extract features. Liu presented within-component and between-component discriminant analysis [16] and color-feature dual discriminating correlation analysis [17] methods, which apply discriminant analysis to enhance the class separability not only within each color component but also between different components. Xiang et al. [18] designed a color two-dimensional principal component analysis method to combine the spatial and color information for color face recognition. Lu et al. [19] presented a color component fusion method using jointly dimension reduction algorithms to select more features from reliable and discriminative components. Zou et al. [20] developed two representation-based classification methods, which model each color image as a quaternionic signal to preserve the color structures of face images. Wu et al. [21] designed two uncorrelated multi-set feature learning methods to extract discriminant features from three color components and reduce the global statistical and discriminating feature-level correlation between components in a multi-set manner. 
(2) A few color face recognition methods extract nonlinear features from color images. For instance, Z. Liu and C. Liu designed a discrete cosine features method [22], which fuses the complementary features derived from discrete cosine transform of color component images in YIQ space. And then they developed a robust face recognition method using color information [23], where three image encoding methods are designed for nonlinear feature extraction in a $\mathrm{RC}_{\mathrm{r}} \mathrm{Q}$ space, and the weighted sum rule is employed to fuse the similarity matrices generated using the features of $\mathrm{R}, \mathrm{C}_{\mathrm{r}}$ and $\mathrm{Q}$ component images. Lajevardi and $\mathrm{Wu}$ [24] built a tensor perceptual color framework for facial expression recognition, which unfolds the color components of face images to 2-D tensors and applies Log-Gabor filters to extract features. Choi et al. [25] presented two color local texture feature methods to exploit the discriminating information achieved from the spatiochromatic texture patterns of different color components within a certain local face region. Elbouz et al. [26] designed a two-level correlation method. In the first level, the face image is decomposed using the RGB or HSV space, and three color components are separately processed and merge through correlations; in the second level, the color-based contour information is converted into a signature, and then the signatures are fused to fabricate one correlator. Taigman et al. [27] developed a nine-layer deep neural network named DeepFace, which is trained on a very large labeled dataset of color face images to obtain a very compact yet sparse face representation. Joshi and Gangwar [28] combined multiscale color local phase quantization features in frequency domain and multiscale color local binary pattern features in spatial domain by using a score level fusion scheme. 
The above color face recognition methods exist following drawbacks:

(a) The methods of first type use the linear transformation or linear projection techniques to extract features, but they do not make full use of the nonlinear characteristics of color face images, such as skin texture, face contours, poses and illuminations.

(b) Among the methods of second type, some methods (e.g., those in Refs. [22, 24-28]) extract nonlinear features from the color component fused images or separately extract nonlinear features from diverse color component images by using the same feature extraction method (that equates to employing the same nonlinear characteristic), but they do not analyze the different nonlinear characteristics of diverse components, respectively; other methods (e.g., that in Ref. [23]) separately extract different nonlinear features from diverse color components, but they do not reduce the between-component correlation, so that this correlation may led to information redundancy and further produce negative influence on recognition results.

To solve the above problems, in this paper, we try to develop a more effective nonlinear feature extraction approach for color face recognition. The contributions are summarized as follows:

We propose a novel dual multi-kernel discriminant analysis (DMDA) approach for color face recognition. In DMDA, we firstly design a kernel selection strategy to select the optimal kernel mapping function for each color component of face images, which can compare different nonlinear characteristics of each color component and choose the most suitable characteristic; then we design a color space selection strategy 
to select the most appropriate color space; finally we separately map different color components of face images into different high-dimensional kernel spaces, and perform multi-kernel learning and discriminant analysis not only within each color component but also between different components. In the nonlinear kernel spaces, DMDA can enhance the within-component and between-component class separabilities, retain the favorable within-class similarity and eliminate the disadvantageous between-class similarity between different color components in the feature level.

We use the public face recognition grand challenge (FRGC) version 2 [29] and labeled faces in the wilds (LFW) [30] databases to evaluate the proposed DMDA approach. Experimental results illustrate that the recognition performance of DMDA is better than that of several representative color face recognition methods.

The rest of this paper is organized as follows. In Section 2, we introduce several representative color face recognition methods and multi-kernel discriminant analysis methods. In Section 3, we propose the DMDA approach. Experimental results are reported in Section 4, and conclusions are drawn in Section 5.

\section{Related Work}

\subsection{Representative Color Face Recognition Methods}

Many color face recognition methods have been presented in the past twenty years. In this paper, we compare our DMDA approach with eight representative color face recognition methods, that is, sparse tensor discriminant color space (STDCS) [9], 
quaternion-based improved locality preserving projection (QILPP) [14], within-component and between-component discriminant analysis (WBDA) [16], quaternion sparse representation-based classification (QSRC) [20], multi-set discriminating uncorrelated projection analysis (MDUPA) [21], color local binary pattern (CLBP) [25], DeepFace (DF) [27] and multiscale color local phase quantization plus multiscale color local binary pattern (MS-CLPQ+MS-CLBP, further abbreviated to DMSC) [28]. Among these methods, STDCS, QILPP, WBDA, QSRC and MDUPA use linear feature extraction techniques, and other methods employ nonlinear techniques; WBDA and MDUPA are most related to our approach, and we detail these two methods as follows.

\subsubsection{Within-component and Between-component Discriminant Analysis (WBDA)}

The objective function of WBDA [16] is defined as

$$
\max _{v_{R}, v_{G}, v_{B}} \frac{S_{b}^{R}+S_{b}^{G}+S_{b}^{B}+\alpha\left(S_{b}^{R G}+S_{b}^{G B}+S_{b}^{B R}\right)}{S_{w}^{R}+S_{w}^{G}+S_{w}^{B}+\alpha\left(S_{w}^{R G}+S_{w}^{G B}+S_{w}^{B R}\right)},
$$

where $v_{i}(i=R, G, B)$ denotes the projective vector of $i$ color component, $S_{b}^{i}$ and $S_{w}^{i}$ separately denote the between-class scatter and within-class scatter of projected features within $i$ component, $S_{b}^{i j}$ and $S_{w}^{i j}(j=R, G, B ; j \neq i)$ separately denote the between-class scatter and within-class scatter of projected features between $i$ and $j$ components, and $\alpha>0$ is a weight coefficient.

\subsubsection{Multi-set Discriminating Uncorrelated Projection Analysis (MDUPA)}

The objective function of MDUPA [21] is defined as 


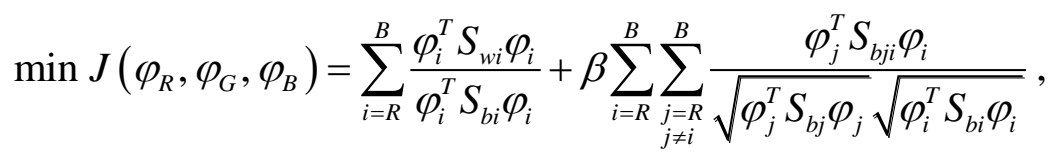

where $\varphi_{i}(i=R, G, B)$ denotes the projective vector of $i$ color component, $S_{b i}$ and $S_{w i}$ separately denote the between-class scatter and within-class scatter matrices of $i$ component, $S_{b j i}(j=R, G, B ; j \neq i)$ denote the between-component between-class scatter matrix of $i$ and $j$ components, and $\beta>0$ is a balance factor.

\subsection{Multi-kernel Discriminant Analysis Methods}

Discriminant analysis is a widely-used dimensionality reduction and feature extraction technique in the field of pattern recognition. The classical linear discriminant analysis (LDA) [31] method uses the Fisher criterion to calculate the optimal projective vectors by maximizing the between-class scatter and simultaneously minimizing the within-class scatter. Many methods have been designed to improve the performance of LDA, such as cost-sensitive semi-supervised discriminant analysis [32], sparse tensor discriminant analysis [33], sparse regularization discriminant analysis [34], generalized quadratic discriminant analysis [35] and incremental semi-supervised discriminant analysis [36].

As a nonlinear technique, kernel learning is widely used. Generalized discriminant analysis [37] is a representative nonlinear discriminant analysis method using the kernel trick, which maps samples into a high-dimensional kernel space and then extracts discriminating features from the kernel mapped samples. In recent years, multi-kernel learning has become a hot topic. It can improve the performance of kernel learning by studying the combination of multiple kernels. Some methods based 
on both multi-kernel learning and discriminant analysis have been presented, such as non-sparse multiple kernel fisher discriminant analysis [38], multiple kernel local Fisher discriminant analysis [39], multiple kernel discriminant analysis in wireless sensor networks [40], two-stage multiple kernel learning for supervised dimensionality reduction [41] and discriminative multiple kernel learning [42].

\section{Dual Multi-kernel Discriminant Analysis (DMDA)}

The notations used in our approach are shown as follows:

$X$ : $\quad$ Training sample set of color face images;

$n: \quad$ Number of all samples in $X$;

$c: \quad$ Number of all classes in $X$;

$n_{p}: \quad$ Number of all samples from $p^{\text {th }}$ class in $X$, where $p=1,2, \cdots, c$;

$N$ : Number of all color components in a color space;

$X_{i}: \quad i^{\text {th }}$ color component sample set of $X$, where $i=1,2, \cdots, N$;

$x_{t}^{i}: \quad t^{\text {th }}$ sample in $X_{i}$, which is a real vector;

$x_{p q}^{i}: \quad q^{\text {th }}$ sample from $p^{\text {th }}$ class in $X_{i}$, which is a real vector;

$y: \quad$ A query sample of color face images;

$y_{i}: \quad i^{\text {th }}$ color component sample of $y$, which is a real vector;

$\phi_{i}$ : Nonlinear kernel mapping of $i^{\text {th }}$ color component;

$k_{i}$ : $\quad$ Kernel function corresponding to $\phi_{i}$;

$K_{i}: \quad$ Kernel matrix of $X_{i}$, whose element in $s^{\text {th }}$ row and $t^{\text {th }}$ column $K_{s t}^{i}=k_{i}\left(x_{s}^{i}, x_{t}^{i}\right)$ 
$K_{y_{i}}$ : Kernel matrix of $y_{i}$, which is a vector with the element in $s^{\text {th }}$ row $K_{s}^{y_{i}}=k_{i}\left(x_{s}^{i}, y_{i}\right)$

$\omega_{i}$ : Ensemble coefficient corresponding to $k_{i}$ for multi-kernel learning, where $\sum_{i=1}^{N} \omega_{i}=1$

$V_{i}: \quad$ Projective transformation of $i^{\text {th }}$ color component, where $V_{i}$ consists of $d$ projective vectors;

$A_{i}$ : $\quad$ Coefficient matrix corresponding to $V_{i}$;

$S_{B}^{i}: \quad$ Between-class scatter of $\phi_{i}\left(X_{i}\right)$

$S_{W}^{i}: \quad$ Within-class scatter of $\phi_{i}\left(X_{i}\right)$

$S_{b}^{i}: \quad$ Between-class scatter matrix of projected features within $\phi_{i}\left(X_{i}\right)$

$S_{w}^{i}: \quad$ Within-class scatter matrix of projected features within $\phi_{i}\left(X_{i}\right)$

$S_{b}^{i j}: \quad$ Between-class scatter matrix of projected features between $\phi_{i}\left(X_{i}\right)$ and $\phi_{j}\left(X_{j}\right)$, where $i \neq j$

$S_{w}^{i j}$ : Within-class scatter matrix of projected features between $\phi_{i}\left(X_{i}\right)$ and $\phi_{j}\left(X_{j}\right)$, where $i \neq j$

$S_{b}^{i \omega}: \quad S_{b}^{i}$ with ensemble coefficients;

$S_{w}^{i \omega}: \quad S_{w}^{i}$ with ensemble coefficients;

$S_{b}^{i j \omega}: \quad S_{b}^{i j} \quad$ with ensemble coefficients;

$S_{w}^{i j \omega}: \quad S_{w}^{i j}$ with ensemble coefficients.

\subsection{Kernel Selection Strategy}

We design the kernel selection strategy as follows: 


$$
\begin{aligned}
& k_{i}^{*}=\underset{k_{i}}{\arg \max _{k_{i}}} S_{k_{i}}, \\
& \text { s.t. } \quad \operatorname{rank}\left(K_{i}\right)=n
\end{aligned}
$$

where $k_{i}$ denotes a kernel (a kernel function with certain parameter values) used for $X_{i}$

$$
S_{k_{i}}=\frac{S_{B}^{i}}{S_{W}^{i}}
$$

denotes the class separability score corresponding to $k_{i}$,

$$
\begin{aligned}
S_{B}^{i} & =\frac{1}{n^{2}-\sum_{p=1}^{c} n_{p}^{2}} \sum_{p=1}^{c} \sum_{q=1}^{c} \sum_{r=1}^{n_{p}} \sum_{t=1}^{n_{q}}\left[\phi_{i}\left(x_{p r}^{i}\right)-\phi_{i}\left(x_{q t}^{i}\right)\right]^{T}\left[\phi_{i}\left(x_{p r}^{i}\right)-\phi_{i}\left(x_{q t}^{i}\right)\right] \\
& =\frac{2}{n^{2}-\sum_{p=1}^{c} n_{p}^{2}}\left[\sum_{p=1}^{c} \sum_{r=1}^{n_{p}}\left(n-n_{p}\right) k_{i}\left(x_{p r}^{i}, x_{p r}^{i}\right)-\sum_{\substack { p=1 \\
\begin{subarray}{c}{q=1 \\
q \neq p{ p = 1 \\
\begin{subarray} { c } { q = 1 \\
q \neq p } }\end{subarray}}^{c} \sum_{r=1}^{n_{p}} \sum_{t=1}^{n_{q}} k_{i}\left(x_{p r}^{i}, x_{q t}^{i}\right)\right], \\
S_{W}^{i} & =\frac{1}{\sum_{p=1}^{c} n_{p}^{2}} \sum_{p=1}^{c} \sum_{r=1}^{n_{p}} \sum_{t=1}^{n_{p}}\left[\phi_{i}\left(x_{p r}^{i}\right)-\phi_{i}\left(x_{p t}^{i}\right)\right]^{T}\left[\phi_{i}\left(x_{p r}^{i}\right)-\phi_{i}\left(x_{p t}^{i}\right)\right] \\
& =\frac{2}{\sum_{p=1}^{c} n_{p}^{2}}\left[\sum_{p=1}^{c} \sum_{r=1}^{n_{p}} n_{p} k_{i}\left(x_{p r}^{i}, x_{p r}^{i}\right)-\sum_{p=1}^{c} \sum_{r=1}^{n_{p}} \sum_{t=1}^{n_{p}} k_{i}\left(x_{p r}^{i}, x_{p t}^{i}\right)\right]
\end{aligned}
$$

and $\operatorname{rank}\left(K_{i}\right)$ denotes the rank of matrix $K_{i}$.

This strategy maximizes the class separability of $\phi_{i}\left(X_{i}\right)$. It should be noted that the constraint $\operatorname{rank}\left(K_{i}\right)=n$ in Formula (3) is used to avoid similar samples in $\phi_{i}\left(X_{i}\right)$, because $n=\operatorname{rank}\left(K_{i}\right)=\operatorname{rank}\left(\left[\phi_{i}\left(X_{i}\right)\right]^{T} \phi_{i}\left(X_{i}\right)\right) \Leftrightarrow \operatorname{rank}\left(\phi_{i}\left(X_{i}\right)\right)=n$. By using this strategy, we can compare different nonlinear characteristics (corresponding to different kernels) of each color component, and select the most suitable nonlinear characteristic. 


\subsection{Color Space Selection Strategy}

In real-world applications, there are many color spaces (or color configurations).

To select an optimum color space for following feature extraction, we use the optimal kernels to calculate the average class separability score

$$
S=\frac{1}{N} \sum_{i=1}^{N} S_{k_{i}^{*}}
$$

for each color space. The optimal color space is the one with maximal score.

This strategy chooses the color space with maximal discriminative ability (corresponding to maximal score) in the kernel space, which is usually considered to be advantageous for following feature extraction. It should be noted that the discriminative ability is measured without employing the between-component class separability scores, because samples of different color components are separately mapped into different kernel spaces and the class separability score between samples in different kernel spaces cannot be calculated.

\subsection{Objective Function of DMDA}

DMDA maps each color components of face images into the selected kernel space. In these kernel spaces, the dimensionalities of color component samples are very high, and thus further feature extraction and dimensionality reduction are demanded.

According to the kernel reproducing theory [43], the projective transformation $V_{i}$ in the kernel space can be linearly expressed by $\phi_{i}\left(X_{i}\right)$ :

$$
V_{i}=\phi_{i}\left(X_{i}\right) A_{i}
$$


Thus the projected feature set of $\phi_{i}\left(X_{i}\right)$

$$
Z_{i}=V_{i}^{T} \phi_{i}\left(X_{i}\right)=A_{i}^{T}\left[\phi_{i}\left(X_{i}\right)\right]^{T} \phi_{i}\left(X_{i}\right)=A_{i}^{T} K_{i} .
$$

Then we separately define $S_{w}^{i}, S_{b}^{i}, S_{w}^{i j}$ and $S_{b}^{i j}$ as

$$
\begin{aligned}
& S_{w}^{i}=\frac{1}{\sum_{p=1}^{c} n_{p}^{2}} \sum_{p=1}^{c} \sum_{r=1}^{n_{p}} \sum_{t=1}^{n_{p}}\left[V_{i}^{T} \phi_{i}\left(x_{p r}^{i}\right)-V_{i}^{T} \phi_{i}\left(x_{p t}^{i}\right)\right]\left[V_{i}^{T} \phi_{i}\left(x_{p r}^{i}\right)-V_{i}^{T} \phi_{i}\left(x_{p t}^{i}\right)\right]^{T} \\
& =\frac{1}{\sum_{p=1}^{c} n_{p}^{2}} V_{i}^{T} \phi_{i}\left(X_{i}\right)\left[\sum_{p=1}^{c} \sum_{r=1}^{n_{p}} \sum_{t=1}^{n_{p}}\left(e_{p r}-e_{p t}\right)\left(e_{p r}-e_{p t}\right)^{T}\right]\left[\phi_{i}\left(X_{i}\right)\right]^{T} V_{i}, \\
& =\frac{2}{\sum_{p=1}^{c} n_{p}^{2}} A_{i}^{T} K_{i}(F-M) K_{i} A_{i} \\
& S_{b}^{i}=\frac{1}{n^{2}-\sum_{p=1}^{c} n_{p}^{2}} \sum_{p=1}^{c} \sum_{\substack{q=1 \\
q \neq p}}^{c} \sum_{r=1}^{n_{p}} \sum_{t=1}^{n_{q}}\left[V_{i}^{T} \phi_{i}\left(x_{p r}^{i}\right)-V_{i}^{T} \phi_{i}\left(x_{q t}^{i}\right)\right]\left[V_{i}^{T} \phi_{i}\left(x_{p r}^{i}\right)-V_{i}^{T} \phi_{i}\left(x_{q t}^{i}\right)\right]^{T} \\
& =\frac{1}{n^{2}-\sum_{p=1}^{c} n_{p}^{2}} V_{i}^{T} \phi_{i}\left(X_{i}\right)\left[\sum_{\substack{c \\
p=1}}^{c} \sum_{\substack{q=1 \\
q \neq p}}^{n_{p}} \sum_{r=1}^{n_{q}} \sum_{t=1}\left(e_{p r}-e_{q t}\right)\left(e_{p r}-e_{q t}\right)^{T}\right]\left[\phi_{i}\left(X_{i}\right)\right]^{T} V_{i}, \\
& =\frac{2}{n^{2}-\sum_{p=1}^{c} n_{p}^{2}} A_{i}^{T} K_{i}\left[\left(n I_{n}-F\right)-\left(E_{n}-M\right)\right] K_{i} A_{i} \\
& S_{w}^{i j}=\frac{1}{\sum_{p=1}^{c} n_{p}^{2}} \sum_{p=1}^{c} \sum_{r=1}^{n_{p}} \sum_{t=1}^{n_{p}}\left[V_{i}^{T} \phi_{i}\left(x_{p r}^{i}\right)-V_{j}^{T} \phi_{j}\left(x_{p t}^{j}\right)\right]\left[V_{i}^{T} \phi_{i}\left(x_{p r}^{i}\right)-V_{j}^{T} \phi_{j}\left(x_{p t}^{j}\right)\right]^{T} \\
& =\frac{1}{\sum_{p=1}^{c} n_{p}^{2}} \sum_{p=1}^{c} \sum_{r=1}^{n_{p}} \sum_{t=1}^{n_{p}}\left\{V_{i}^{T} \phi_{i}\left(X_{i}\right) e_{p r} e_{p r}^{T}\left[\phi_{i}\left(X_{i}\right)\right]^{T} V_{i}-V_{i}^{T} \phi_{i}\left(X_{i}\right) e_{p r} e_{p t}^{T}\left[\phi_{j}\left(X_{j}\right)\right]^{T} V_{j}\right. \\
& \left.-V_{j}^{T} \phi_{j}\left(X_{j}\right) e_{p t} e_{p r}^{T}\left[\phi_{i}\left(X_{i}\right)\right]^{T} V_{i}+V_{j}^{T} \phi_{j}\left(X_{j}\right) e_{p t} e_{p t}^{T}\left[\phi_{j}\left(X_{j}\right)\right]^{T} V_{j}\right\} \\
& =\frac{1}{\sum_{p=1}^{c} n_{p}^{2}}\left(A_{i}^{T} K_{i} F K_{i} A_{i}-A_{i}^{T} K_{i} M K_{j} A_{j}-A_{j}^{T} K_{j} M K_{i} A_{i}+A_{j}^{T} K_{j} F K_{j} A_{j}\right)
\end{aligned}
$$




$$
\begin{gathered}
S_{b}^{i j}=\frac{1}{n^{2}-\sum_{p=1}^{c} n_{p}^{2}} \sum_{\substack { p=1 \\
\begin{subarray}{c}{q=1 \\
q \neq p{ p = 1 \\
\begin{subarray} { c } { q = 1 \\
q \neq p } }\end{subarray}}^{c} \sum_{r=1}^{n_{p}} \sum_{t=1}^{n_{q}}\left[V_{i}^{T} \phi_{i}\left(x_{p r}^{i}\right)-V_{j}^{T} \phi_{j}\left(x_{q t}^{j}\right)\right]\left[V_{i}^{T} \phi_{i}\left(x_{p r}^{i}\right)-V_{j}^{T} \phi_{j}\left(x_{q t}^{j}\right)\right]^{T} \\
=\frac{1}{n^{2}-\sum_{p=1}^{c} n_{p}^{2}} \sum_{\substack { p=1 \\
\begin{subarray}{c}{q=1 \\
q \neq p{ p = 1 \\
\begin{subarray} { c } { q = 1 \\
q \neq p } }\end{subarray}}^{c} \sum_{r=1}^{n_{p}} \sum_{t=1}^{n_{q}}\left\{V_{i}^{T} \phi_{i}\left(X_{i}\right) e_{p r} e_{p r}^{T}\left[\phi_{i}\left(X_{i}\right)\right]^{T} V_{i}\right. \\
\quad-V_{i}^{T} \phi_{i}\left(X_{i}\right) e_{p r} e_{q t}^{T}\left[\phi_{j}\left(X_{j}\right)\right]^{T} V_{j}-V_{j}^{T} \phi_{j}\left(X_{j}\right) e_{q t} e_{p r}^{T}\left[\phi_{i}\left(X_{i}\right)\right]^{T} V_{i},(1) \\
\left.\quad+V_{j}^{T} \phi_{j}\left(X_{j}\right) e_{q t} e_{q t}^{T}\left[\phi_{j}\left(X_{j}\right)\right]^{T} V_{j}\right\} \\
=\frac{1}{n^{2}-\sum_{p=1}^{c} n_{p}^{2}}\left[A_{i}^{T} K_{i}\left(n I_{n}-F\right) K_{i} A_{i}-A_{i}^{T} K_{i}\left(E_{n}-M\right) K_{j} A_{j}\right. \\
\left.-A_{j}^{T} K_{j}\left(E_{n}-M\right) K_{i} A_{i}+A_{j}^{T} K_{j}\left(n I_{n}-F\right) K_{j} A_{j}\right]
\end{gathered}
$$

where $e_{p r}=[\underbrace{0, \cdots, 0}_{\sum_{q=1}^{p-1} n_{q}+r-1}, 1, \underbrace{0, \cdots, 0}_{\sum_{q=p+1}^{c} n_{q}+n_{p}-r}]^{T} \in \mathbf{R}^{n}$,

$$
M=\left[\begin{array}{cccc}
E_{n_{1}} & 0 & \cdots & 0 \\
0 & E_{n_{2}} & \cdots & 0 \\
\vdots & \vdots & \cdots & \vdots \\
0 & 0 & \cdots & E_{n_{c}}
\end{array}\right] \in \mathbf{R}^{n \times n}, \quad F=\left[\begin{array}{cccc}
n_{1} I_{n_{1}} & 0 & \cdots & 0 \\
0 & n_{2} I_{n_{2}} & \cdots & 0 \\
\vdots & \vdots & \cdots & \vdots \\
0 & 0 & \cdots & n_{c} I_{n_{c}}
\end{array}\right] \in \mathbf{R}^{n \times n},
$$

$E_{n_{p}}$ denotes a $n_{p} \times n_{p}$ matrix whose elements are all equal to $1, I_{n_{p}}$ denotes a $n_{p} \times n_{p}$ identity matrix, $E_{n}$ denotes a $n \times n$ matrix whose elements are all equal to 1 , and $I_{n}$ denotes a $n \times n$ identity matrix. It should be noted that $S_{w}^{i j}=S_{w}^{j i}$ and $S_{b}^{i j}=S_{b}^{j i}$

Further we add the ensemble coefficients $\omega_{1}, \omega_{2}, \cdots, \omega_{N}$ to $S_{w}^{i}, S_{b}^{i}, S_{w}^{i j}$ and $S_{b}^{i j}$ for multi-kernel learning via replacing $K_{i}$ by $\omega_{i} K_{i}$ (which is equivalent to replacing $k_{i}$ by $\omega_{i} k_{i}$ ), and obtain

$$
S_{w}^{i \omega}=\frac{2 \omega_{i}^{2}}{\sum_{p=1}^{c} n_{p}^{2}} A_{i}^{T} K_{i}(F-M) K_{i} A_{i}
$$




$$
\begin{aligned}
& S_{b}^{i \omega}=\frac{2 \omega_{i}^{2}}{n^{2}-\sum_{p=1}^{c} n_{p}^{2}} A_{i}^{T} K_{i}\left[\left(n I_{n}-F\right)-\left(E_{n}-M\right)\right] K_{i} A_{i}, \\
& S_{w}^{i j \omega}=\frac{1}{\sum_{p=1}^{c} n_{p}^{2}}\left(\omega_{i}^{2} A_{i}^{T} K_{i} F K_{i} A_{i}-\omega_{i} \omega_{j} A_{i}^{T} K_{i} M K_{j} A_{j}\right. \\
& S_{b}^{i j \omega}=\frac{1}{n^{2}-\sum_{p=1}^{c} n_{p}^{2}}\left[\omega_{i}^{2} A_{i}^{T} K_{i}\left(n I_{n}-F\right) K_{i}^{T} A_{j} M K_{i} A_{i}+\omega_{i}^{2} A_{j}^{T} K_{j} A_{i}^{T} K_{j} A_{j}\right) \\
& \quad-\omega_{j} \omega_{i} A_{j}^{T} K_{j}\left(E_{n}-M\right) K_{j} A_{j}
\end{aligned}
$$

Here $S_{w}^{i j \omega}=S_{w}^{j i \omega}$ and $S_{b}^{i j \omega}=S_{b}^{j i \omega}$.

Finally, to enhance the within-component and between-component class separabilities, retain the favorable within-class similarity and reduce the disadvantageous between-class similarity between color components in the feature level, we define the objective function of DMDA as

$$
\begin{array}{ll}
\max _{\substack{A_{1}, A_{2}, \cdots, A_{N} \\
\omega_{1}, \omega_{2}, \cdots, \omega_{N}}} \frac{\operatorname{tr}\left(\sum_{i=1}^{N} S_{b}^{i \omega}+\sum_{i=1}^{N} \sum_{\substack{j=1 \\
j \neq i}}^{N} S_{b}^{i j \omega}\right)}{\operatorname{tr}\left(\sum_{i=1}^{N} S_{w}^{i \omega}+\sum_{i=1}^{N} \sum_{\substack{j=1 \\
j \neq i}}^{N} S_{w}^{i j \omega}\right),} \\
\text { s.t. } \quad \sum_{i=1}^{N} \omega_{i}=1
\end{array}
$$

where $\operatorname{tr}(\cdot)$ denotes the trace of a square matrix.

When $A_{1}, A_{2}, \cdots, A_{N}$ and $\omega_{1}, \omega_{2}, \cdots, \omega_{N}$ are achieved, we can obtain the final features of the training sample set $X$ and the query sample $y$ by

$$
\begin{aligned}
Z_{X} & =\left[\left(A_{1}^{T} K_{1}\right)^{T},\left(A_{2}^{T} K_{2}\right)^{T}, \cdots,\left(A_{N}^{T} K_{N}\right)^{T}\right]^{T}, \\
Z_{y} & =\left[\left(A_{1}^{T} K_{y_{1}}\right)^{T},\left(A_{2}^{T} K_{y_{2}}\right)^{T}, \cdots,\left(A_{N}^{T} K_{y_{N}}\right)^{T}\right]^{T} .
\end{aligned}
$$


And then we can classify $y$ based on $X$ by using $Z_{y}, Z_{X}$ and the nearest neighbor classifier.

\subsection{Algorithm Description for Solution of Objective Function}

Formula (18) can be divided into the following two sub-problems:

$$
\begin{aligned}
& \max _{A} \frac{\operatorname{tr}\left(A^{T} L A\right)}{\operatorname{tr}\left(A^{T} J A\right)} \quad \text { (when } \omega_{1}, \omega_{2}, \cdots, \omega_{N} \text { are given), } \\
& \max _{\omega} \frac{\omega^{T} P \omega}{\omega^{T} Q \omega} \quad \text { (when } A_{1}, A_{2}, \cdots, A_{N} \text { are given), } \\
& \text { s.t. } \omega^{T} e_{N}=1
\end{aligned}
$$

where $e_{N} \in \mathbf{R}^{N}$ with all elements equal to 1 ,

$$
\begin{aligned}
& A=\left[\begin{array}{c}
A_{1} \\
A_{2} \\
\vdots \\
A_{N}
\end{array}\right], \omega=\left[\begin{array}{c}
\omega_{1} \\
\omega_{2} \\
\vdots \\
\omega_{N}
\end{array}\right], \quad L=\left[\begin{array}{cccc}
\omega_{1}^{2} K_{1} L_{1} K_{1} & \omega_{1} \omega_{2} K_{1} L_{2} K_{2} & \cdots & \omega_{1} \omega_{N} K_{1} L_{2} K_{N} \\
\omega_{2} \omega_{1} K_{2} L_{2} K_{1} & \omega_{2}^{2} K_{2} L_{1} K_{2} & \cdots & \omega_{2} \omega_{N} K_{2} L_{2} K_{N} \\
\vdots & \vdots & \vdots & \vdots \\
\omega_{N} \omega_{1} K_{N} L_{2} K_{1} & \omega_{N} \omega_{2} K_{N} L_{2} K_{2} & \cdots & \omega_{N}^{2} K_{N} L_{1} K_{N}
\end{array}\right] \text {, } \\
& J=\left[\begin{array}{cccc}
\omega_{1}^{2} K_{1} J_{1} K_{1} & \omega_{1} \omega_{2} K_{1} J_{2} K_{2} & \cdots & \omega_{1} \omega_{N} K_{1} J_{2} K_{N} \\
\omega_{2} \omega_{1} K_{2} J_{2} K_{1} & \omega_{2}^{2} K_{2} J_{1} K_{2} & \cdots & \omega_{2} \omega_{N} K_{2} J_{2} K_{N} \\
\vdots & \vdots & \vdots & \vdots \\
\omega_{N} \omega_{1} K_{N} J_{2} K_{1} & \omega_{N} \omega_{2} K_{N} J_{2} K_{2} & \cdots & \omega_{N}^{2} K_{N} J_{1} K_{N}
\end{array}\right], \\
& P=\left[\begin{array}{cccc}
\operatorname{tr}\left(A_{1}^{T} K_{1} L_{1} K_{1} A_{1}\right) & \operatorname{tr}\left(A_{1}^{T} K_{1} L_{2} K_{2} A_{2}\right) & \cdots & \operatorname{tr}\left(A_{1}^{T} K_{1} L_{2} K_{N} A_{N}\right) \\
\operatorname{tr}\left(A_{2}^{T} K_{2} L_{2} K_{1} A_{1}\right) & \operatorname{tr}\left(A_{2}^{T} K_{2} L_{1} K_{2} A_{2}\right) & \cdots & \operatorname{tr}\left(A_{2}^{T} K_{2} L_{2} K_{N} A_{N}\right) \\
\vdots & \vdots & \vdots & \vdots \\
\operatorname{tr}\left(A_{N}^{T} K_{N} L_{2} K_{1} A_{1}\right) & \operatorname{tr}\left(A_{N}^{T} K_{N} L_{2} K_{2} A_{2}\right) & \cdots & \operatorname{tr}\left(A_{N}^{T} K_{N} L_{1} K_{N} A_{N}\right)
\end{array}\right], \\
& Q=\left[\begin{array}{cccc}
\operatorname{tr}\left(A_{1}^{T} K_{1} J_{1} K_{1} A_{1}\right) & \operatorname{tr}\left(A_{1}^{T} K_{1} J_{2} K_{2} A_{2}\right) & \cdots & \operatorname{tr}\left(A_{1}^{T} K_{1} J_{2} K_{N} A_{N}\right) \\
\operatorname{tr}\left(A_{2}^{T} K_{2} J_{2} K_{1} A_{1}\right) & \operatorname{tr}\left(A_{2}^{T} K_{2} J_{1} K_{2} A_{2}\right) & \cdots & \operatorname{tr}\left(A_{2}^{T} K_{2} J_{2} K_{N} A_{N}\right) \\
\vdots & \vdots & \vdots & \vdots \\
\operatorname{tr}\left(A_{N}^{T} K_{N} J_{2} K_{1} A_{1}\right) & \operatorname{tr}\left(A_{N}^{T} K_{N} J_{2} K_{2} A_{2}\right) & \cdots & \operatorname{tr}\left(A_{N}^{T} K_{N} J_{1} K_{N} A_{N}\right)
\end{array}\right], \\
& L_{1}=4\left(n I_{n}-F\right)+2\left(M-E_{n}\right), \quad L_{2}=M-E_{n}, \quad J_{1}=4 F-2 M, \text { and } J_{2}=-M \text {. }
\end{aligned}
$$

To solve Formula (18), we design an iterative algorithm as follows: 


\section{Algorithm 1.}

Step 1. Let $\omega_{i}=1 / N(i=1,2, \cdots, N)$ and $v_{1}=-1000$.

Step 2. Calculate $A_{1}, A_{2}, \cdots, A_{N}$ by using Formula (21). When the eigenvectors $\alpha^{1}, \alpha^{2}, \cdots, \alpha^{d}$ corresponding to the $d$ largest eigenvalues of $J^{-1} L$ are achieved, let $A=\left[\alpha^{1}, \alpha^{2}, \cdots, \alpha^{d}\right]$. We can obtain $A_{1}, A_{2}, \cdots, A_{N}$ from $A$.

Step 3. Let $v_{2}=\operatorname{tr}\left(A^{T} L A\right) / \operatorname{tr}\left(A^{T} J A\right)$. If $v_{2}-v_{1} \leq \varepsilon \quad(\varepsilon>0$ is a threshold $)$, stop the iteration and output $A_{1}, A_{2}, \cdots, A_{N}, \omega_{1}, \omega_{2}, \cdots, \omega_{N}$; otherwise, let $v_{1}=v_{2}$ and go to Step 4.

Step 4. Calculate $\omega_{1}, \omega_{2}, \cdots, \omega_{N}$ by using Formula (22). Once the eigenvector $\omega$ corresponding to the largest eigenvalue of $Q^{-1} P$ are obtained, let $\omega=\omega /\left(\omega^{T} e_{N}\right)$, and we can get $\omega_{1}, \omega_{2}, \cdots, \omega_{N}$ from $\omega$. Go to Step 2 .

\section{Experiments}

\subsection{Experimental Database Introduction}

The public face recognition grand challenge (FRGC) version 2 database [29] consists of controlled and uncontrolled color face images. The controlled images show good image quality, while the uncontrolled images are taken under complex background and thus display poor image quality. In Experiment 4 of FRGC database, we select 222 individuals with 36 images per person from the training set to construct our experimental database (named FRGC-OE database), where 18 controlled images and 18 uncontrolled images are chosen for each individual. We perform the rotation and scaling transformations to align the centers of two eyes to the predefined locations, 
crop a facial region sub-image, and resize the sub-image to $60 \times 60$. Fig. 2 (a) shows all demo images of an individual in FRGC-OE database.

The labeled faces in the wilds (LFW) database [30] contains several preprocessed sets. In the deep funneled image set, we select 106 persons who hold more than 14 images, and choose 14 images per person to construct our experimental database (called LFW-OE database). For each selected image, we crop a facial region sub-image and resize the sub-image to $60 \times 60$. Fig. 2 (b) shows all demo images of an individual in LFW-OE database.

In FRGC-OE and LFW-OE databases, we randomly select 8 images per person for training and use the remainder for testing.

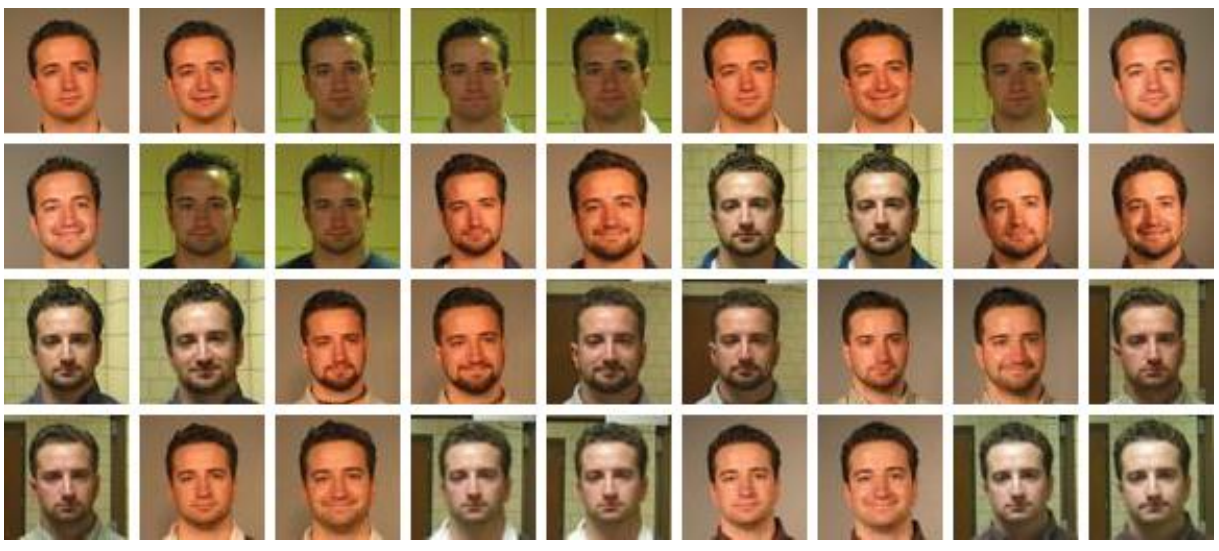

(a) In FRGC-OE database.

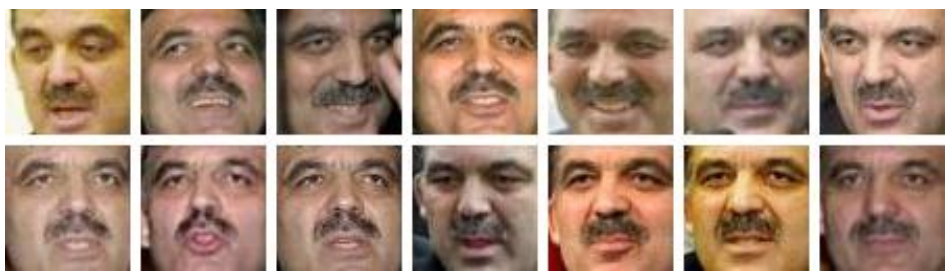

(b) In LFW-OE database.

Fig. 2. Demo images of an individual. 


\subsection{Experiment for Kernel Selection}

To select the optimal kernel for each color component of face images, we firstly use Formula (4) to calculate the class separability score of each kernel, and then obtain the optimal kernel by using Formula (3). We make the kernel selection experiment with the linear kernel (LK, as Formula (23)), polynomial kernel (PK, as Formula (24)), Gaussian kernel (GK, as Formula (25)), Cauchy kernel (CK, as Formula (26)), inverse multi-quadric kernel (MK, as Formula (27)) and generalized t-student kernel (TK, as Formula (28)) in five common color spaces (that is, RGB, HSV, $\mathrm{YC}_{\mathrm{b}} \mathrm{C}_{\mathrm{r}}$, YIQ and CIE-Luv) and two hybrid color spaces (i.e. normalized ZRG and $\mathrm{RQC}_{\mathrm{r}}$ [25]), where CIE-Luv and normalized ZRG are hereinafter abbreviated to Luv and ZRG, respectively.

$$
\begin{aligned}
& k(x, y)=x^{T} y, \\
& k(x, y)=\left(a x^{T} y+b\right)^{p}, \\
& k(x, y)=\exp \left(-\|x-y\|^{2} / \sigma\right), \\
& k(x, y)=1 /\left(1+\|x-y\|^{2} / \sigma\right), \\
& k(x, y)=1 / \sqrt{\|x-y\|^{2}+c}, \\
& k(x, y)=1 /\left(1+\|x-y\|^{q}\right),
\end{aligned}
$$

where $x$ and $y$ denote two sample vectors, $a, b, c, p, q$ and $\sigma$ are adjustable parameters. It should be noted that for a kernel (a kernel function with certain parameter values), we calculate the average class separability score across 10 calculations with random training sample selection. Table 1 and Table 2 separately show the selected kernel and corresponding class separability score of each color component in the FRGC-OE and LFW-OE databases. 
Table 1. Selected kernel and corresponding class separability score of each color component in FRGC-OE database.

Selected kernel name (selected parameter values) and corresponding class separability score

\begin{tabular}{lccc} 
Color space & $1^{\text {st }}$ component & $2^{\text {nd }}$ component & $3^{\text {rd }}$ component \\
RGB & LK & LK & LK \\
& 1.41 & 1.36 & 1.31 \\
HSV & GK $\left(\sigma=2.96 \times 10^{8}\right)$ & LK & LK \\
& 1.44 & 1.22 & 1.30 \\
YC $_{b} C_{r}$ & LK & GK $\left(\sigma=3.56 \times 10^{11}\right)$ & GK $\left(\sigma=2.75 \times 10^{11}\right)$ \\
& 1.26 & 1.33 & 1.43 \\
YIQ & LK & GK $\left(\sigma=1.08 \times 10^{7}\right)$ & GK $\left(\sigma=4.90 \times 10^{5}\right)$ \\
& 1.46 & 1.44 & 1.36 \\
Luv & LK & LK & GK $\left(\sigma=6.59 \times 10^{10}\right)$ \\
& 1.43 & 1.34 & 1.22 \\
ZRG & LK & GK $\left(\sigma=2.58 \times 10^{7}\right)$ & GK $\left(\sigma=9.13 \times 10^{6}\right)$ \\
& 1.37 & 1.35 & 1.27 \\
RQC & LK & GK $\left(\sigma=4.90 \times 10^{5}\right)$ & GK $\left(\sigma=2.75 \times 10^{11}\right)$ \\
& 1.41 & 1.36 & 1.43 \\
\hline
\end{tabular}

Table 2. Selected kernel and corresponding class separability score of each color component in LFW-OE database.

\begin{tabular}{|c|c|c|c|}
\hline \multirow[b]{2}{*}{ Color space } & \multicolumn{3}{|c|}{$\begin{array}{c}\text { Selected kernel name (selected parameter values) } \\
\text { and corresponding class separability score }\end{array}$} \\
\hline & $1^{\text {st }}$ component & $2^{\text {nd }}$ component & $3^{\text {rd }}$ component \\
\hline \multirow[t]{2}{*}{ RGB } & $\mathrm{PK}\left(a=10^{-8}, b=10^{-8}, p=2\right)$ & $\mathrm{PK}\left(a=10^{-8}, b=5 \times 10^{-8}, p=2\right)$ & LK \\
\hline & 1.53 & 1.45 & 1.46 \\
\hline \multirow[t]{2}{*}{ HSV } & $\operatorname{GK}\left(\sigma=1.04 \times 10^{6}\right)$ & PK ( & $\left.a=10^{-8}, b=5 \times 10^{-8}, p=2\right)$ \\
\hline & 1.17 & 1.38 & 1.53 \\
\hline \multirow[t]{2}{*}{$\mathrm{YC}_{\mathrm{b}} \mathrm{C}_{\mathrm{r}}$} & $\mathrm{PK}\left(a=10^{-12}, b=10^{-7}, p=2\right)$ & LK & GK $\left(\sigma=5.01 \times 10^{11}\right)$ \\
\hline & 1.49 & 1.32 & 1.35 \\
\hline \multirow[t]{2}{*}{ YIQ } & $\mathrm{PK}\left(a=10^{-8}, b=10^{-8}, p=2\right)$ & $\operatorname{GK}\left(\sigma=1.57 \times 10^{7}\right)$ & LK \\
\hline & 1.49 & 1.34 & 1.37 \\
\hline \multirow[t]{2}{*}{ Luv } & $\mathrm{PK}\left(a=10^{-11}, b=0.07, p=3\right)$ & PK ( & $\left(a=10^{-10}, b=10^{-7}, p=2\right)$ \\
\hline & 1.50 & 1.32 & 1.32 \\
\hline \multirow[t]{2}{*}{ ZRG } & LK & $\operatorname{GK}\left(\sigma=3.53 \times 10^{7}\right)$ & LK \\
\hline & 1.46 & 1.33 & 1.34 \\
\hline \multirow[t]{2}{*}{$\mathrm{RQC}_{\mathrm{r}}$} & $\mathrm{PK}\left(a=10^{-8}, b=10^{-8}, p=2\right)$ & LK & $\operatorname{GK}\left(\sigma=5.01 \times 10^{11}\right)$ \\
\hline & 1.53 & 1.37 & 1.35 \\
\hline
\end{tabular}




\subsection{Experiment for Color Space Selection}

According to Table 1 and Table 2, we calculate the average class separability score for each color space, which is shown in Table 3. It is clear that the average class separability score of YIQ and RGB color spaces are maximal in the FRGC-OE and LFW-OE databases, respectively. Thus we separately adopt the YIQ and RGB spaces in the FRGC-ours and LFW-ours databases for our DMDA approach in the following recognition experiment.

Table 3. Average class separability scores of seven color spaces.

\begin{tabular}{lll}
\hline & \multicolumn{2}{l}{ Average class separability score } \\
Color space & FRGC-OE & LFW-OE \\
RGB & 1.36 & $\mathbf{1 . 4 8}$ \\
$\mathrm{HSV}$ & 1.32 & 1.36 \\
$\mathrm{YC}_{\mathrm{b}} \mathrm{C}_{\mathrm{r}}$ & 1.34 & 1.39 \\
$\mathrm{YIQ}$ & $\mathbf{1 . 4 2}$ & 1.40 \\
$\mathrm{Luv}$ & 1.33 & 1.38 \\
$\mathrm{ZRG}$ & 1.33 & 1.38 \\
$\mathrm{RQC}_{\mathrm{r}}$ & 1.40 & 1.42 \\
\hline
\end{tabular}

\subsection{Recognition Experiment of DMDA Approach}

We compare the proposed DMDA approach with eight representative color face recognition methods including STDCS [9], QILPP [14], WBDA [16], QSRC [20], MDUPA [21], CLBP [25], DF [27] and DMSC [28]. In the experiment, for our DMDA approach, we use YIQ color space in FRGC-OE database and RGB space in LFW-OE database; for STDCS, QILPP, WBDA, QSRC, MDUPA, DF and DMSC, we employ RGB space in accordance with their references; for CLBP, we adopt ZRG and $\mathrm{RQC}_{\mathrm{r}}$ spaces according to Ref. [25]. By employing the cross validation technique, we 
set $\varepsilon$ in Algorithm 1 as 0.1 , and set the number of projective vectors $d$ in the obtained projective transformations as 258 in FRGC-OE database and 273 in LFW-OE database. The parameter values of other compared methods are also determined by using the cross validation technique. At the classification stage, we employ the nearest neighbor classifier for all compared methods except for QSRC, and QSRC itself is a classifier.

In FRGC-OE and LFW-OE databases, we run all compared methods 20 times with random training and testing sample selection. Fig. 3 shows the recognition rates of all compared methods across 20 runs in two databases. Table 4 shows the corresponding means and standard deviations of recognition rates. We can see that as compared with other methods, DMDA improves the average recognition rates at least by $1.43 \%(=84.65 \%-83.22 \%)$ in FRGC-OE database and $3.24 \%(=69.51 \%-66.27 \%)$ in LFW-OE database.

Table 4 also reports the average training and testing time of all compared methods, where "no" denotes no training time, the CPU of computer is Intel Core2 E7500, and the RAM memory capacity is 4GB. As shown in Table 4, the training time of DMDA is less than that of other iterative training methods including STDCS and DF, although DMDA spends more training time than four non-iterative training methods including QILPP, WBDA, MDUPA and CLBP; the testing time of all compared methods except for QSRC is directly influenced by the final feature number and feature extraction of the testing sample; QSRC spends much more testing time than other methods, because it directly performs classification without training. 


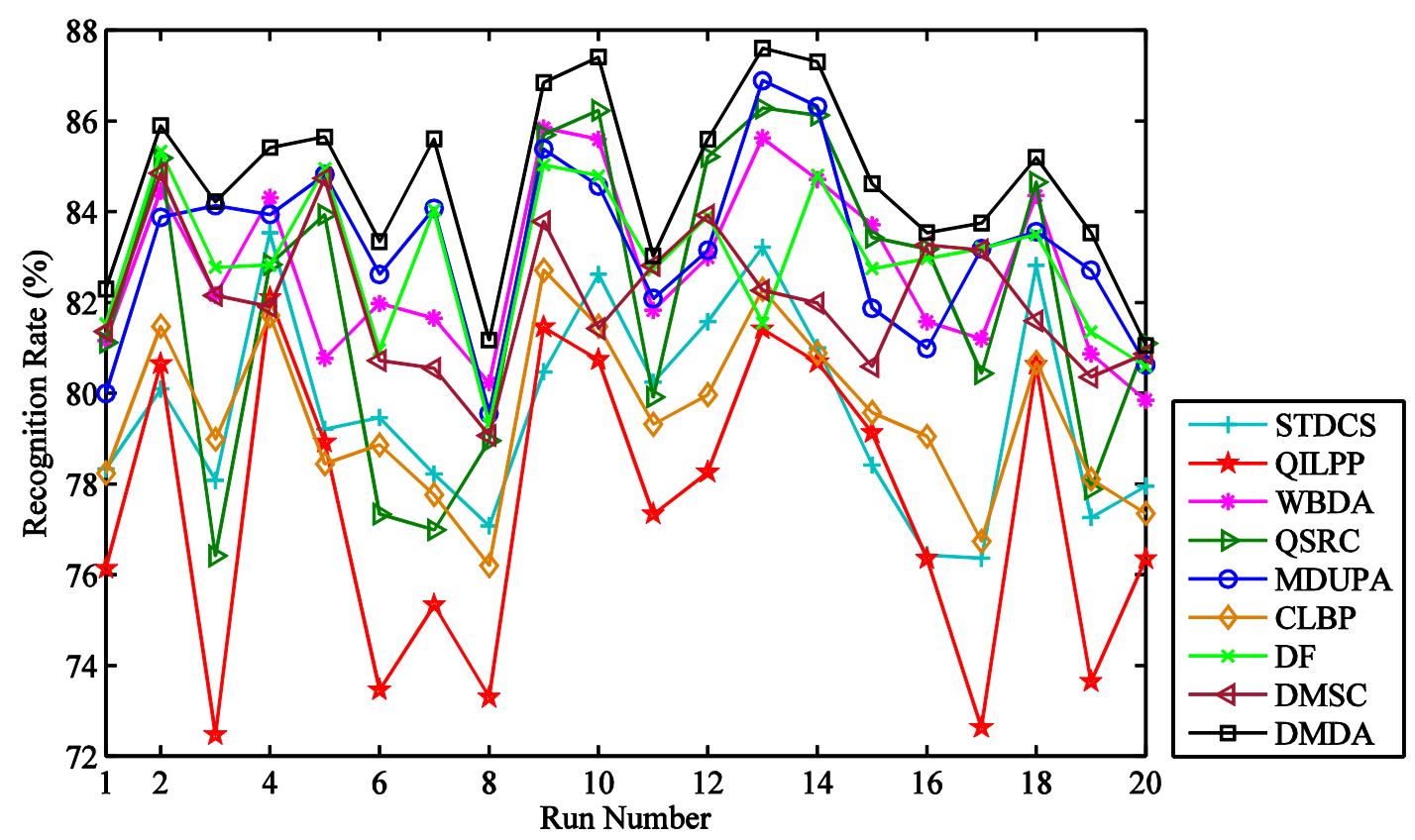

(a) In FRGC-OE database.

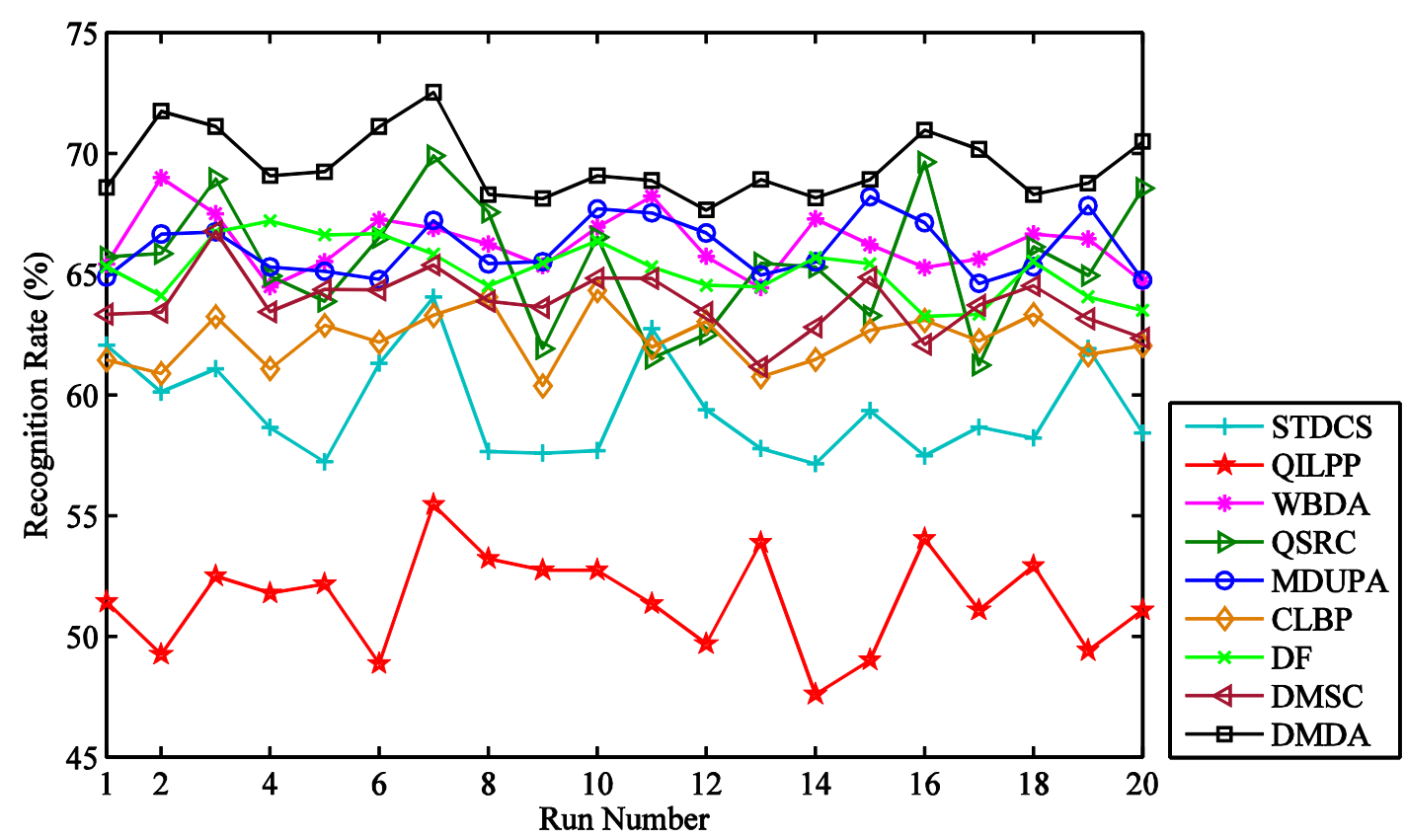

(b) In LFW-OE database.

Fig. 3. Recognition rates of all compared methods. 
Table 4. Means and standard deviations of recognition rates and average training and testing time.

\begin{tabular}{lllll}
\hline & \multicolumn{2}{c}{ Recognition rate $(\%)$} & \multicolumn{2}{l}{ Average training/testing time (second) } \\
Method & FRGC-OE & LFW-OE & FRGC-OE & LFW-OE \\
STDCS & $79.62 \pm 2.26$ & $59.43 \pm 2.07$ & $580.85 / 0.0260$ & $206.60 / 0.0086$ \\
QILPP & $77.55 \pm 3.28$ & $51.52 \pm 2.03$ & $129.22 / 0.0748$ & $27.88 / 0.0060$ \\
WBDA & $82.74 \pm 1.92$ & $66.27 \pm 1.23$ & $95.35 / 0.0126$ & $16.53 / 0.0040$ \\
QSRC & $82.15 \pm 3.36$ & $65.52 \pm 2.61$ & no/979.8425 & no/458.5812 \\
MDUPA & $83.22 \pm 1.98$ & $66.10 \pm 1.20$ & $95.67 / 0.0125$ & $18.89 / 0.0039$ \\
CLBP & $79.49 \pm 1.86$ & $62.31 \pm 1.11$ & $193.24 / 0.0658$ & $66.25 / 0.0632$ \\
DF & $82.94 \pm 1.67$ & $65.20 \pm 1.19$ & $638.34 / 0.0853$ & $304.76 / 0.0575$ \\
DMSC & $82.07 \pm 1.55$ & $63.82 \pm 1.25$ & $482.21 / 0.0981$ & $136.98 / 0.0959$ \\
DMDA & $\mathbf{8 4 . 6 5} \pm \mathbf{1 . 9 4}$ & $\mathbf{6 9 . 5 1} \pm \mathbf{1 . 3 7}$ & $\mathbf{3 5 1 . 4 2 / 0 . 0 1 4 5}$ & $\mathbf{8 9 . 2 4 / 0 . 0 1 0 4}$ \\
\hline
\end{tabular}

To evaluate the statistical significance of difference between DMDA and other compared methods, we conduct the Mcnemar's test [44], where the performance difference between two compared methods is considered to be statistically significant if the p-value is below 0.05. Table 5 shows the p-values between DMDA and other compared methods in the FRGC-OE and LFW-OE databases. It is clear that DMDA makes a statistically significant difference in comparison with other methods.

Table 5. P-values between DMDA and other compared methods.

\begin{tabular}{cll}
\hline & \multicolumn{2}{c}{ P-value } \\
Other compared method & FRGC-OE & LFW-OE \\
STDCS & $5.88 \times 10^{-11}$ & $2.43 \times 10^{-15}$ \\
QILPP & $3.06 \times 10^{-11}$ & $4.44 \times 10^{-19}$ \\
WBDA & $1.68 \times 10^{-7}$ & $1.35 \times 10^{-8}$ \\
QSRC & $2.97 \times 10^{-4}$ & $2.82 \times 10^{-8}$ \\
MDUPA & $2.53 \times 10^{-7}$ & $3.78 \times 10^{-8}$ \\
CLBP & $7.66 \times 10^{-14}$ & $7.15 \times 10^{-14}$ \\
DF & $9.71 \times 10^{-6}$ & $3.10 \times 10^{-9}$ \\
DMSC & $6.59 \times 10^{-6}$ & $2.66 \times 10^{-12}$ \\
\hline
\end{tabular}




\subsection{Validation Experiment for Kernel Selection Strategy}

In this subsection, we compare the recognition rates of the proposed DMDA approach using the optimal kernel (i.e. the optimal kernel function with optimal parameter values) and the worst kernel (i.e. the worst kernel function with optimal parameter values) in YIQ color space of FRGC-OE database and RGB space of LFW-OE database.

Table 6 shows the parameter setting of DMDA using the worst kernel, where "YIQ" denotes YIQ space of FRGC-OE database and "RGB" denotes RGB space of LFW-OE database. Fig. 4 shows the recognition rates of DMDA using the optimal and worst kernels across 20 runs in two databases, and Table 7 shows corresponding means and standard deviations of recognition rates, where "DMDA-opt" denotes DMDA using the optimal kernel, and "DMDA-wor" denotes DMDA using the worst kernel. We can find that DMDA using the optimal kernel significantly outperforms that using the worst kernel.

Table 6. Parameter setting of DMDA using the worst kernel.

\begin{tabular}{cccccc}
\hline \multicolumn{7}{l}{ Worst kernel with corresponding class separability score } \\
YIQ & $1^{\text {st }}$ component & $2^{\text {nd }}$ component & $3^{\text {rd }}$ component & $\varepsilon$ & $d$ \\
& TK $(q=0.1)$ & TK $(q=0.1)$ & TK $(q=0.1)$ & 0.1 & 443 \\
RGB & TK $(q=0.1)$ & TK $(q=0.1)$ & TK $(q=0.1)$ & & \\
& 1.15 & 1.15 & 1.15 & 1.15 & 126 \\
\hline
\end{tabular}




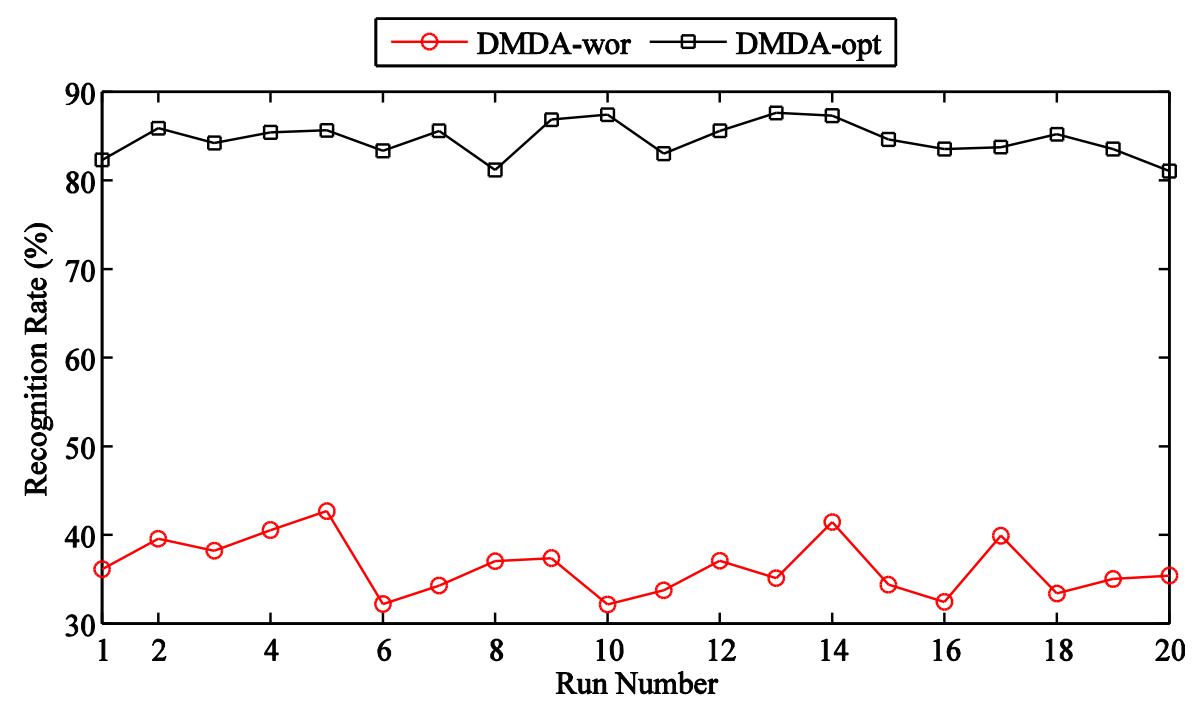

(a) In FRGC-OE database.

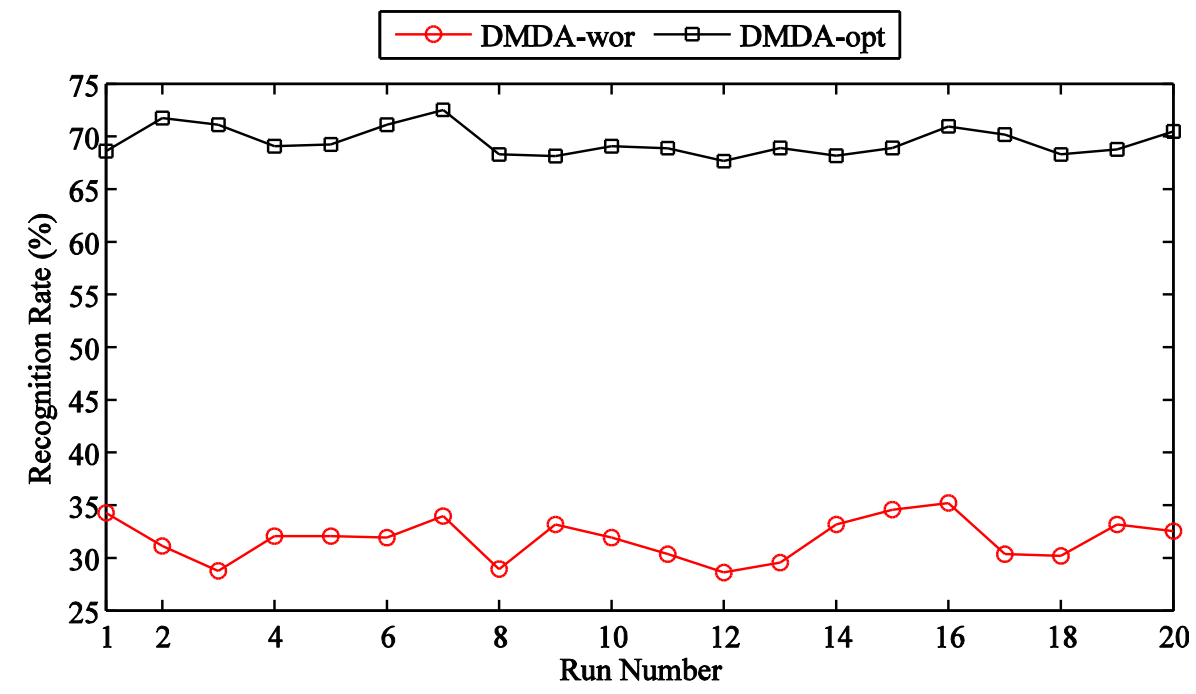

(b) In LFW-OE database.

Fig. 4. Recognition rates of DMDA using the optimal and worst kernels.

Table 7. Means and standard deviations of recognition rates.

\begin{tabular}{lll}
\hline & \multicolumn{2}{c}{ Recognition rate (\%) } \\
Method & FRGC-OE & LFW-OE \\
DMDA-wor & $36.41 \pm 3.17$ & $31.80 \pm 2.01$ \\
DMDA-opt & $\mathbf{8 4 . 6 5} \pm \mathbf{1 . 9 4}$ & $\mathbf{6 9 . 5 1} \pm \mathbf{1 . 3 7}$ \\
\hline
\end{tabular}




\subsection{Validation Experiment for Color Space Selection Strategy}

In this subsection, we compare the recognition rates of DMDA using the optimal kernel in the RGB, HSV, $\mathrm{YC}_{\mathrm{b}} \mathrm{C}_{\mathrm{r}}$, YIQ, Luv, ZRG and $\mathrm{RQC}_{\mathrm{r}}$ color spaces.

Table 8 shows the parameter setting of DMDA using seven spaces, except for the setting of kernel functions and their parameters, which has been shown in Section 4.2. Fig. 5 shows the recognition rates of DMDA using seven spaces across 20 runs in FRGC-OE and LFW-OE databases, and Table 9 shows corresponding means and standard deviations of recognition rates. It is obvious that DMDA using YIQ space in FRGC-OE database or using RGB space in LFW-OE database remarkably outperforms that using other spaces, and the fluctuation of average recognition rates in Table 9 is similar to that of average class separability scores in Table 3, except for the score fluctuation of $\mathrm{YC}_{\mathrm{b}} \mathrm{C}_{\mathrm{r}}$ and Luv spaces in LFW-OE database.

Table 8. Parameter setting of DMDA using seven color spaces.

\begin{tabular}{lllll}
\hline & \multicolumn{2}{l}{ FRGC-OE } & \multicolumn{2}{l}{ LFW-OE } \\
Color space & $\varepsilon$ & $d$ & $\varepsilon$ & $d$ \\
RGB & 0.1 & 443 & 0.1 & 273 \\
HSV & 0.1 & 221 & 0.1 & 252 \\
YC $_{b} C_{\mathrm{r}}$ & 0.1 & 221 & 0.1 & 105 \\
YIQ & 0.1 & 258 & 0.1 & 126 \\
Luv & 0.1 & 221 & 0.1 & 126 \\
ZRG & 0.1 & 221 & 0.1 & 168 \\
RQC $_{\mathrm{r}}$ & 0.1 & 258 & 0.1 & 168 \\
\hline
\end{tabular}




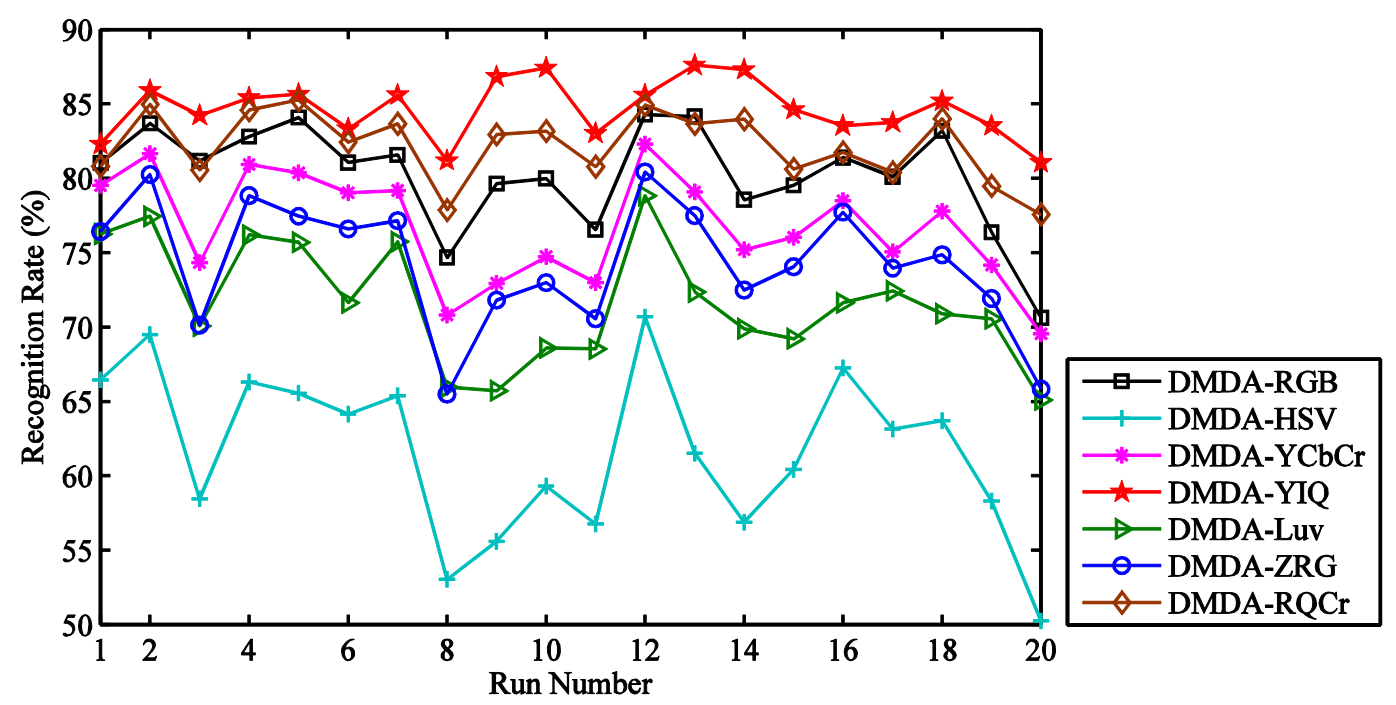

(a) In FRGC-OE database.

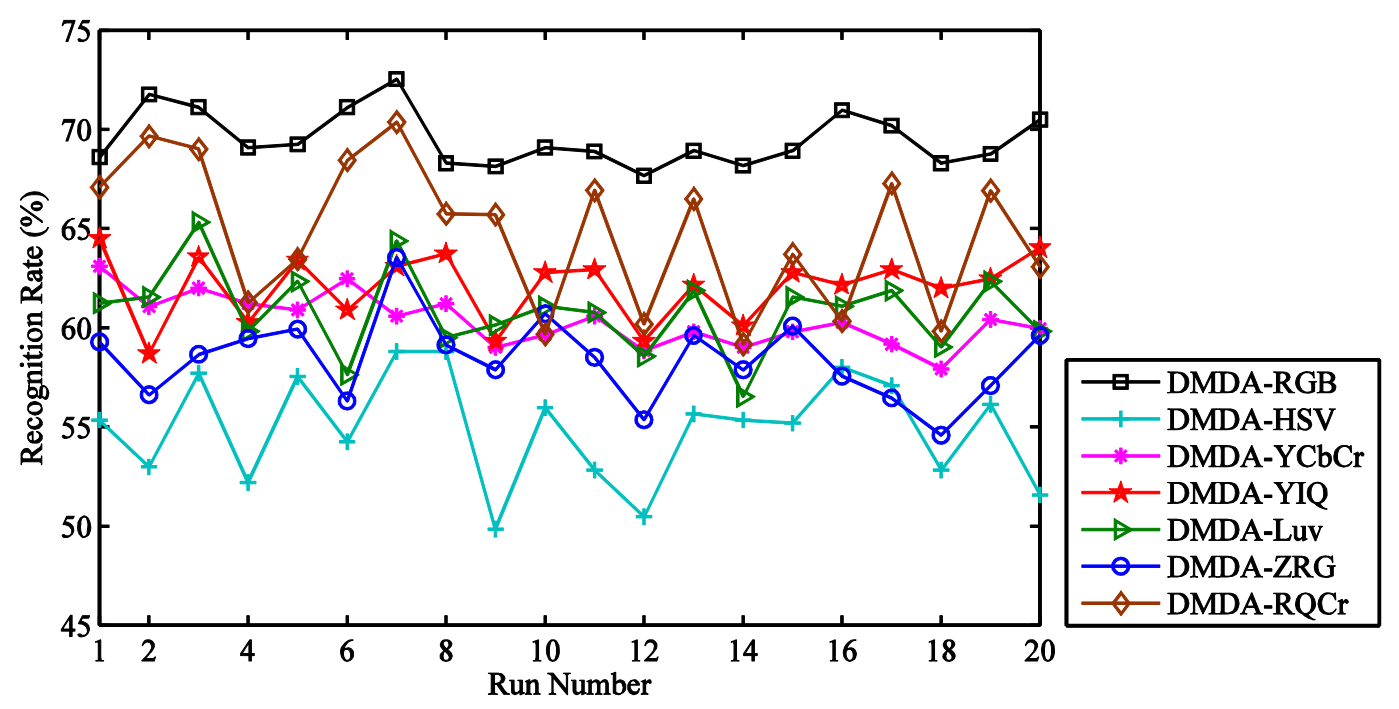

(b) In LFW-OE database.

Fig. 5. Recognition rates of DMDA using seven color spaces.

Table 9. Means and standard deviations of recognition rates.

\begin{tabular}{lll}
\hline & \multicolumn{2}{c}{ Recognition rate (\%) } \\
Method & FRGC-OE & LFW-OE \\
DMDA-RGB & $80.23 \pm 3.52$ & $\mathbf{6 9 . 5 1} \pm \mathbf{1 . 3 7}$ \\
DMDA-HSV & $61.63 \pm 5.51$ & $54.93 \pm 2.71$ \\
DMDA-YC $_{b} C_{\mathrm{r}}$ & $76.71 \pm 3.64$ & $60.35 \pm 1.29$ \\
DMDA-YIQ & $\mathbf{8 4 . 6 5} \pm \mathbf{1 . 9 4}$ & $62.06 \pm 1.71$ \\
DMDA-Luv & $71.64 \pm 4.00$ & $60.83 \pm 2.08$ \\
DMDA-ZRG & $74.32 \pm 4.24$ & $58.42 \pm 2.06$ \\
DMDA-RQC $_{\mathrm{r}}$ & $82.17 \pm 2.32$ & $64.72 \pm 3.65$ \\
\hline
\end{tabular}




\subsection{Further Analysis}

In this subsection, we show the class separabilities of final training sample features for all compared methods (except for QSRC, because QSRC does not extract features of training samples), which are usually measured by the Fisher value [31]:

$$
S_{F}=\frac{\operatorname{tr}\left(S_{B}^{F}\right)}{\operatorname{tr}\left(S_{W}^{F}\right)},
$$

where $S_{B}^{F}$ and $S_{W}^{F}$ denote the between-class and within-class scatter matrices of the final training sample features, respectively.

It should be noted that the tensor-based method, i.e. STDCS, needs to acquire sample feature vectors at first. Let $A \in \mathbf{R}^{L_{1} \times L_{2} \times L_{3}}$ denotes a projected tensor in these methods. We divide $A$ into $L_{3}$ second-order tensors $A_{1}, A_{2}, \cdots, A_{L_{3}} \in \mathbf{R}^{L_{1} \times L_{2}}$, use the vector form to represent these second-order tensors, and concatenate the $L_{3}$ vectors into one augmented vector.

Table 10. Average Fisher values of all compared methods (except for QSRC).

\begin{tabular}{lll}
\hline & \multicolumn{2}{c}{ Average Fisher value } \\
Method & FRGC-OE & LFW-OE \\
STDCS & 0.93 & 0.45 \\
QILPP & 0.78 & 0.28 \\
WBDA & 1.17 & 0.73 \\
MDUPA & 1.36 & 0.70 \\
CLBP & 0.91 & 0.52 \\
DF & 1.26 & 0.64 \\
DMSC & 1.10 & 0.57 \\
DMDA & $\mathbf{2 . 0 4}$ & $\mathbf{0 . 8 9}$ \\
\hline
\end{tabular}

Table 10 shows the average Fisher values of all compared methods (except for QSRC) across 20 runs in FRGC-OE and LFW-OE databases. It is clear that DMDA 
achieves maximal Fisher value, and the fluctuation of the average Fisher values is similar to that of the average recognition rates in Table 4 , which illustrates that the class separabilities of final training sample features directly influence the recognition results.

\section{Conclusions}

In this paper, we propose a novel nonlinear feature extraction approach for color face recognition, called dual multi-kernel discriminant analysis (DMDA). In the nonlinear kernel spaces, DMDA can enhance the within-component and between-component class separabilities, maintain the favorable within-class similarity and reduce the disadvantageous between-class similarity between color components in the feature level. Experimental results in the FRGC version 2 and LFW databases show that the recognition performance of our approach outperforms that of several representative color face recognition methods including STDCS, QILPP, WBDA, QSRC, MDUPA, CLBP, DF and DMSC, the kernel selection and color space selection strategies of DMDA are reasonable and effective, and the recognition results are directly affected by the class separabilities of final training sample features.

\section{Acknowledgment}

The work described in this paper was fully supported by the National Natural

Science Foundation of China under Project No. 61601229, the Natural Science Foundation of JiangSu Province (Youth Fund) under Project No. BK20160966, the 
Natural Science Foundation of the Jiangsu Higher Education Institutions of China under Project No. 16KJB510022 and A Project Funded by the Priority Academic Program Development of Jiangsu Higher Education Institutions.

The face images we used come from the public face recognition grand challenge (FRGC) version 2 [29] and labeled faces in the wilds (LFW) [30] databases. The description of FRGC database is shown on https://www.nist.gov/programsprojects/face-recognition-grand-challenge-frgc, and we obtained the FRGC database by following the instructions for the FRGC license on https://sites.google.com/a /nd.edu/public-cvrl/data-sets. The details of LFW database is shown on http://viswww.cs.umass.edu/lfw/, where we downloaded the deep funneled image set.

\section{References}

[1] J. Yang, C. Liu, What Kind of Color Spaces is Suitable for Color Face Recognition?, Neurocomputing 73(10-12) (2010) 2140-2146.

[2] R. Niese, A. Al-Hamadi, A. Farag, H. Neumann, B. Michaelis, Facial expression recognition based on geometric and optical flow features in colour image sequences, IET Computer Vision, $6(2)(2012) 79-89$.

[3] P. Zhao, Robust wood species recognition using variable color information, Optik 124(17) (2013) 2833-2836.

[4] Z. Liu, C. Liu, Fusion of color, local spatial and global frequency information for face recognition, Pattern Recognition, 43(8) (2010) 2882-2890.

[5] J.Y. Choi, Y.M. Ro, K.N. Plataniotis, A comparative study of preprocessing mismatch effects in color image based face recognition, Pattern Recognition, 44(2) (2011) 412-430.

[6] C. Liu, Extracting Discriminative Color Features for Face Recognition, Pattern Recognition Letters 32(14) (2011) 1796-1804. 
[7] J.Y. Choi, Y. M. Ro, K. N. Plataniotis, Boosting color feature selection for color face recognition, IEEE Trans. Image Processing 20(5) (2011) 1425-1434.

[8] S. Wang, J. Yang, N. Zhang, C. Zhou, Tensor discriminant color space for face recognition, IEEE Trans. Image Processing 20(9) (2011) 2490-2501.

[9] S. Wang, J. Yang, M. Sun, X. Peng, M. Sun, C. Zhou, Sparse tensor discriminant color space for face verification, IEEE Trans. Neural Networks and Learning Systems 23(6) (2012) 876-888.

[10] W.J. Shin, S.H. Lee, H.S. Min, H. Sohn, Y.M. Ro, Face verification using color sparse representation, Lecture Notes in Computer Science (Int. Workshop on Digital Forensics and Watermaking) 7809 (2013) 290-299.

[11] C. Liu, Effective use of color information for large scale face verification, Neurocomputing 101 (2013) 43-51

[12] C.R. Zhao, D.Q. Miao, Z.H. Lai, C. Gao, C.C. Liu, J.Y. Yang, Two-dimensional color uncorrelated discriminant analysis for face recognition, Neurocomputing 113 (2013) 251-261.

[13] S.C. Huang, J. Chen, Z. Luo, Sparse tensor CCA for color face recognition, Neural Computing and Applications 24(7-8) (2014) 1647-1658.

[14] S. Wu, Quaternion-based improved LPP method for color face recognition, Optik 125(10) (2014) 2344-2349.

[15] Y.F. Sun, H.J. Jia, Y.L. Hu, B.C. Yin, Color face recognition based on color image correlation similarity discriminant model, Multimedia Tools and Applications, 73(3) (2014) 2063-2079.

[16] Q. Liu, Within-component and between-component discriminant analysis for color face recognition, Optik 125(21) (2014) 6366-6374

[17] Q. Liu, Colour-feature dual discriminating correlation analysis for face recognition, IET Computer Vision 9(4) (2015) 467-475

[18] X.G. Xiang, J. Yang, Q.P. Chen, Color face recognition by PCA-like approach, Neurocomputing $152(2015) 231-235$

[19] Z. Lu, X.D. Jiang, A.C. Kot, A color channel fusion approach for face recognition, IEEE Signal Processing Letters 22(11) (2015) 1839-1843.

[20] C.M. Zou, K.I. Kou, Y.L. Wang, Quaternion collaborative and sparse representation with application to color face recognition, IEEE Trans. Image Processing 25(7) (2016) 3287-3302. 
[21] F. Wu, X.Y. Jing, X.W. Dong, Q. Ge, S.S. Wu, Q. Liu, D. Yue, J.Y. Yang, Uncorrelated multi-set feature learning for color face recognition, Pattern Recognition 60 (2016) 630-646.

[22] Z. Liu, C. Liu, Fusion of the complementary discrete cosine features in the YIQ color space for face recognition, Computer Vision and Image Understanding, 111(3) (2008) 249-262.

[23] Z. Liu, C. Liu, Robust face recognition using color information, Lecture Notes in Computer Science (Int. Conf. Biometrics) 5558 (2009) 122-131.

[24] S.M. Lajevardi, H.R. Wu, Facial expression recognition in perceptual color space, IEEE Trans. Image Processing 21(8) (2012) 3721-3733.

[25] J.Y. Choi, Y.M. Ro, K.N. Plataniotis, Color local texture features for color face recognition, IEEE Trans. Image Processing 21(3) (2012) 1366-1380.

[26] M. Elbouz, A. Alfalou, C. Brosseau, M.S. Alam, S. Qasmi, Correlation-based efficient face recognition and color change detection, Optics Communications, 311(2) (2013) 186-200.

[27] Y. Taigman, M. Yang, M. Ranzato, L. Wolf, DeepFace: closing the gap to human-level performance in face verification, in Proc. IEEE Conf. Computer Vision and Pattern Recognition, 2014, pp. 1701-1708.

[28] A. Joshi, A.K. Gangwar, Color local phase quantization (CLPQ)- a new face representation approach using color texture cues, in Proc. Int. Conf. Biometrics, 2015, pp. 177-184.

[29] P.J. Phillips, P.J. Flynn, T. Scruggs, K. Bowyer, J. Chang, K. Hoffman, J. Marques, J. Min, W. Worek, Overview of the face recognition grand challenge, in Proc. IEEE Conf. Computer Vision and Pattern Recognition, 2005, pp. 947-954.

[30] G.B. Huang, M. Mattar, H. Lee, E. Learned-Miller, Learning to align from scratch, in Proc. Advances in Neural Information Processing Systems, 2012, pp. 764-772.

[31] A.M. Martinez, A.C. Kak, PCA versus LDA, IEEE Trans. Pattern Analysis and Machine Intelligence, 23(2) (2001) 228-233

[32] J.W. Lu, X.Z. Zhou, Y.P. Tan, Y.Y. Shang, J. Zhou, Cost-sensitive semi-supervised discriminant analysis for face recognition, IEEE Trans. Information Forensics and Security, 7(3) (2012) 944-953

[33] Z.H. Lai, Y. Xu, J. Yang, J.H. Tang, D. Zhang, Sparse tensor discriminant analysis, IEEE Trans. Image Processing, 22(10) (2013) 3904-3915. 
[34] F. Yin, L.C. Jiao, F.H. Shang, L. Xiong, X.D. Wang, Sparse regularization discriminant analysis for face recognition, Neurocomputing, 128 (2014) 341-362.

[35] S. Bose, A. Pal, R. Saharay, J. Nayak, Generalized quadratic discriminant analysis, Pattern Recognition 48(8) (2015) 2676-2684.

[36] T.I. Dhamecha, R. Singh, M. Vatsa, On incremental semi-supervised discriminant analysis, Pattern Recognition 52 (2016) 135-147.

[37] G. Baudat, F. Anouar, Generalized discriminant analysis using a kernel approach, Neural Computation, 12(10) (2000) 2385-2404.

[38] F. Yan, J. Kittler, K. Mikolajczyk, A. Tahir, Non-sparse multiple kernel fisher discriminant analysis, J. Machine Learning Research 13(1) (2012) 607-642.

[39] Z.Q. Wang, X. Sun, Multiple kernel local Fisher discriminant analysis for face recognition, Signal Processing 93(6) (2013) 1496-1509.

[40] X.Z. Liu, G. Yang, Distributed face recognition using multiple kernel discriminant analysis in wireless sensor networks, Int. J. Distributed Sensor Networks 2014(242105) (2014) 1-7.

[41] A. Nazarpour, P. Adibi, Two-stage multiple kernel learning for supervised dimensionality reduction, Pattern Recognition 48(5) (2015) 1854-1862.

[42] Q.W. Wang, Y.F. Gu, D. Tuia, Discriminative multiple kernel learning for hyperspectral image classification, IEEE Trans. Geoscience and Remote Sensing 54(7) (2016) 3912-3927.

[43] J.S. Taylor, N. Cristianini, Kernel methods for pattern analysis, Cambridge University Press, Cambridge, 2004.

[44] W.S. Yambor, B.A. Draper, J.R. Beveridge, Analyzing PCA-based face recognition algorithms: eigenvector selection and distance measures, Empirical Evaluation Methods in Computer Vision (2002) 39-60. 